\title{
Juventud y universidad: sujetos y escenarios para el debate crítico y autorreflexivo sobre el consumo de sustancias psicoactivas de uso legal e ilegal*
}

\section{Youth and University: Subjects and Scenarios for Critical and Self-Reflective Debate on the Use of Legal and Illegal Psychoactive Drugs}

\section{Juventude e universidade: sujeitos e cenários para o debate crítico e autorreflexivo sobre o consumo de substancias psicoativas de uso legal e ilegal}

Fecha de recepción: 01-12-14 Fecha de aceptación: 18-03-15 Disponible en línea: 30-06-15 doi: 10.11144/Javeriana.rgyps18-28.juse

Como citar este artículo:

Calderón-Romero EA, Cáliz-Romero NE. Juventud y universidad: sujetos y escenarios para el debate crítico y autorreflexivo sobre el consumo de sustancias psicoactivas de uso legal e ilegal. Rev. Gerenc. Polit. Salud. 2015; 14(28): 123-141. http://dx.doi.org/10.11144/Javeriana.rgyps18-28.juse

\author{
Eliana Andrea Calderón-Romero** \\ Nelly Esther Cáliz-Romero***
}

Artículo de investigación. Proyecto de investigación: Motivos de un grupo de jóvenes universitarios de los programas académicos de Ciencias de la Salud y Pecuarias de la Universidad de Ciencias Aplicadas y Ambientales que inducen hacia el consumo de sustancias psicoactivas legales e ilegales. Inicio: julio del 2013; finalización: noviembre del 2014. Universidad de Ciencias Aplicadas y Ambientales (UDCA).

* Enfermera de la Universidad de Ciencias Aplicadas y Ambientales (UDCA). Enfermera clínica de la Fundación Hospital San Carlos (FHSC), Bogotá, Colombia. Correo electrónico: elicaro13@hotmail.com. Dirección: Calle 89B No. 116A- 30, interior 28 apto. 101, Bogotá, Colombia.

*** Enfermera de la Universidad Nacional de Colombia, magíster en Piscología Comunitaria, candidata a doctora en Salud Colectiva, docente en categoría de titular de la Facultad de Enfermería en la Universidad de Ciencias Aplicadas y Ambientales (UDCA), Bogotá, Colombia. Correo electrónico: ncaliz@udca.edu.co. 


\section{Resumen}

El objetivo de esta investigación fue la comprensión de los motivos que llevaron a un grupo de jóvenes universitarios a optar por el consumo de sustancias psicoactivas de uso legal e ilegal. Se trató de una investigación cualitativa de estudio de caso, con diseño fenomenológico, en la cual participaron 32 estudiantes. Las técnicas investigativas fueron la observación participante, la observación no participante y los grupos focales. Para el análisis de datos se aplicó la distinción de momentos y reducciones fenomenológicas y criterios de codificación abierta, axial y selectiva. Los principales resultados fueron que las sustancias más consumidas son el alcohol, el tabaco y la marihuana, en tanto que las motivaciones se asocian al ámbito familiar, académico, de la relación entre pares, a la decisión personal, el microtráfico de sustancias de uso ilegal y el comercio de sustancias legales. El consumo de drogas resulta de una red de determinaciones sociales, en relación con la cual, la Universidad podría abrir un debate crítico y autorreflexivo en función del sujeto y no de las sustancias.

Palabras clave: drogas ilícitas; adolescente; determinantes sociales de la salud; universidades; consumidores de drogas; autonomía personal

\section{Abstract}

The purpose of this research was to understand the reasons that led a group of young university students into the use of legal and illegal psychoactive drugs. This is a qualitative case study research, with a phenomenological design, with the participation of 32 students. The research techniques used were participant observation, non-participant observation, and focus groups. For data analysis we applied moment distinction, phenomenological reductions; and open, axial, and selective codification criteria. The main results show that the most used substances are alcohol, tobacco, and marihuana; regarding motivations, these are associated to family and academic settings, relationship with peers, personal decision, micro-traffic of illegal substances, and commerce of legal substances. Drug use turns into a network of social determinations around which the University could open a critical and self-reflective debate, focused on the subject and not on the substances.

Keywords: illegal drugs; teenagers; social determinants of health; universities; drug users; personal autonomy

\section{Resumo}

O objetivo desta pesquisa foi a compreensão das motivações que levaram uma turma de jovens universitários optar pelo consumo de substancias psicoativas de uso legal e ilegal. Tratou-se de pesquisa qualitativa de estudo de caso, com desenho fenomenológico, na qual participaram 32 discentes. As técnicas investigativas foram a observação participante, observação não participante e grupos focais. Para a análise de dados aplicou-se a distinção de momentos e reduções fenomenológicas e critérios de codificação aberta, axial e seletiva. Os principais resultados foram que substancias mais consumidas são álcool, tabaco e maconha, entanto que as motivações associam-se ao âmbito familiar, acadêmico, do relacionamento entre pares, decisão pessoal, microtráfico de substancias de uso ilegal e comercio de substancias legais. O consumo de droga resulta de uma rede de determinações sociais, em relação com a qual, a universidade poderia abrir debate crítico e autorreflexivo em função do sujeito e não das substancias.

Palavras-chave: drogas ilícitas; adolescente; determinantes sociais da saúde; universidades; consumidores de droga; autonomia pessoal 


\section{Introducción}

El consumo de sustancias psicoactivas es una práctica ancestral en la humanidad. Comunidades antiguas del Medio Oriente, Asia, América, Europa y África, así como comunidades indígenas actuales, han utilizado estas sustancias como parte de su medicina tradicional o como medio para transitar al plano espiritual. Sin embargo, en sociedades occidentalizadas, esta práctica ha alcanzado dimensiones sociales totalmente diferentes, generando problemas de salud pública tales como la adicción, la violencia y la propagación del VIH-SIDA.

En el panorama epidemiológico mundial, la tasa de consumo de sustancias de uso legal e ilegal se mantiene estable, ya que el aumento en el número de consumidores es proporcional al crecimiento de la población mundial. En el año 2010 se calcula que unos 230 millones de personas consumieron alguna droga de uso ilícito, por lo menos una vez, y unos 27 millones de personas se consideran consumidores problemáticos, es decir, el equivalente al $0,6 \%$ de la población mundial (1).

En el año 2012, entre 162 y 324 millones de personas consumieron una sustancia de uso ilícito, principalmente sustancias del grupo del Cannabis, los opioides, la cocaína o los estimulantes de tipo anfetamínico, con un rango de edad entre 15 y 64 años. Se reportan alrededor de 16 a 39 millones de consumidores problemáticos, lo que equivale al $0,6 \%$ de la población adulta mundial (2).

En el 2011, el número de muertes relacionadas con el consumo de drogas se estimó en 211000. La mayoría se produjo entre la población más joven de consumidores y en gran medida podía haberse prevenido. Los opioides siguieron siendo el grupo de sustancias implicadas en muertes relacionadas con las drogas, notificado con más frecuencia. En el 2012 se informó de un total aproximado de 183000 muertes relacionadas con las drogas, esa cifra corresponde a una tasa de mortalidad de 40 muertes por millón en la población de entre 15 y 64 años de edad (3).

Según el Estudio Nacional de Consumo de Sustancias Psicoactivas en Colombia, del 2013, 87\% de las personas declaran haber consumido alcohol al menos alguna vez en su vida, siendo en hombres el $91 \%$ y en mujeres el $83 \%$, con una edad de inicio alrededor de 16 años en hombres y 18 años en mujeres. El uso de bebidas alcohólicas se incrementa conforme aumenta el nivel socioeconómico: $32,3 \%$ en el estrato 1 y $42,1 \%$ en los estratos 4,5 y 6 . La mayor tasa de consumo se presenta en jóvenes entre 18 y 24 años, con 49,2\%, y en jóvenes entre 25 y 34 años, con $45,4 \%$. De acuerdo con las cifras del estudio, cerca de 2,6 millones de personas en Colombia con edades entre 12 y 65 consumen alcohol siguiendo un patrón que se puede considerar riesgoso o perjudicial. Esto equivale al $31 \%$ de los consumidores actuales de alcohol y al $11,1 \%$ de la población de 12 a 65 años (4).

En el caso de las sustancias psicoactivas de uso ilegal, la prevalencia en el último año estima que unas 839000 personas usaron al menos una vez, una o más de las siguientes sustancias: marihuana, cocaína, bazuco, éxtasis, heroína, sustancias inhalables o Dick. De estas personas, 667000 son varones y 172000 mujeres. El mayor consumo de sustancias de uso ilícito en el último año se presenta en el grupo de 18 a 24 años, con una tasa del $8,7 \%$, seguido por adolescentes de 12 a 17 años con $4,8 \%$ y personas de 25 a 34 años con una prevalencia de $4,3 \%$ (4).

En cuanto a la marihuana, el 11,5\% de las personas entre 12 y 65 años han probado esta sustancia al menos una vez en la vida, con una tasa tres veces mayor en los hombres que en 
las mujeres y una edad promedio de inicio de consumo de 17,6 años en ambos sexos. Se observa que entre las personas que han consumido marihuana en el último año, más del $57,6 \%$ muestran signos de abuso o dependencia, siendo esta relación mayor en los varones (58\%). Las personas que consumen marihuana y presentan signos y síntomas de uso problemático tienen en su mayoría entre 18 y 24 años. Este grupo etario representa más del $40 \%$ del total de dependientes y el $44 \%$ del total de abusadores (4).

Los jóvenes universitarios en Latinoamérica siguen un patrón similar: la sustancia psicoactiva más consumida de uso legal, es el alcohol, y de uso ilegal, la marihuana. En Colombia, el 95,8\% de los estudiantes declararon haber consumido alcohol alguna vez en la vida, seguido de Ecuador con un $88,7 \%$, Perú con $87,5 \%$ y Bolivia un $77,1 \%$. La edad promedio de inicio del consumo de alcohol es de 17,5 años en Bolivia, 15,3 años en Colombia, 16,4 años en Ecuador y 16,2 años en Perú. Entre los consumidores de alcohol del último año, 39,7\% de los estudiantes de Bolivia, 36,6\% en Ecuador, $31,1 \%$ en Colombia y $25,1 \%$ en Perú califican como consumidores que presentan riesgo o uso perjudicial de alcohol, siendo mayor el porcentaje entre hombres que entre mujeres, en todos los países (5).

Respecto a la marihuana, en Colombia uno de cada tres estudiantes declara haber consumido esta sustancia por lo menos una vez en la vida. En Ecuador este comportamiento llega a $21,3 \%$ y a menos del $12 \%$ en Bolivia y Perú, con una edad promedio de inicio de 18 años. El consumo es mayor en hombres que en mujeres en los cuatro países. La prevalencia del último año de consumo de marihuana presenta tasas de 3,6\% en Bolivia, 15,2\% en Colombia, 8,8\% en Ecuador y 4,2\% en Perú.

126 Es así como Colombia presenta la mayor tasa de consumo de marihuana y, a la vez, los estudiantes colombianos muestran una menor percepción de riesgo con respecto al uso de esta sustancia y mayor acceso a ella. Se estima también que entre 26 y $29 \%$ de los estudiantes de Colombia, Ecuador y Perú pueden ser considerados abusadores o con signos de dependencia a la marihuana (5).

Este panorama nos muestra que la práctica de consumo de sustancias psicoactivas es una situación de alcance local y mundial. Las estadísticas que indican signos de abuso, dependencia y muerte, reflejan una realidad problemática, en razón, primero, a que son seres humanos y, segundo, a una etapa de la vida en la que hombres y mujeres proyectan su existencia.

En el campo de las ciencias de la salud y las ciencias humanas, la evidencia científica muestra que las características que pueden predisponer al consumo o abuso de sustancias psicoactivas de uso legal e ilegal por parte de la población universitaria, son: accesibilidad, disponibilidad, curiosidad, necesidad de evasión de situaciones, necesidad de sentirse aceptado por el grupo, búsqueda de placer, rebeldía y sentido del riesgo (6). También se identifica que un consumo irresponsable de estas sustancias, es resultado del fácil acceso al consumo recreativo, el cual puede conllevar los efectos más nocivos, como bajo autocontrol, dificultad en la toma de decisiones, violencia física y sexual y problemas en las relaciones sociales (7).

Se argumenta también que el consumo de sustancias psicoactivas adquiere un sentido particular en el escenario universitario y en los estudiantes, en tanto facilita el encuentro con el otro, la identificación y diferenciación simultánea, propiciando una desinhibición que lleva al relajamiento, la diversión y la ampliación de los lazos sociales, premisas fundamentales en la experiencia universitaria. Es así como el consumo de este tipo de 
sustancias en la vida universitaria cumple en muchas ocasiones una función que da soporte a una noción de autonomía y adultez y a la respuesta de las exigencias sociales y académicas de un ámbito que resulta nuevo, tentador y amenazante, a la vez (8). La vida universitaria es con frecuencia una cultura en sí misma, con muchas oportunidades para experimentar el consumo de varias sustancias a las cuales no se había estado expuesto anteriormente, como es el caso de "fiestas" desenfrenadas, dentro o fuera de la universidad, y la promoción que se hace del uso del alcohol y otras sustancias para disminuir el estrés y divertirse (9).

Sin embargo, al hacer un razonamiento crítico a este tipo de investigaciones, se identifica que sus abordajes y métodos desarticulan el fenómeno de las drogas del contexto sociopolítico y económico donde se genera, situando el análisis y el debate en los factores predisponentes de sujeto, en muchas ocasiones como atributos individuales. Jáuregui también reconoce esta limitación cuando plantea que el problema de las drogas parece vivir en un limbo social, pues ningún debate incluye la estructura social, política y económica que rodea el fenómeno. Pareciera que la adolescencia por sí sola crea el problema, pocos son los autores e investigadores que ven el problema en todo su contexto, porque ello implicaría poner en tela de juicio todo el entramado social, además del sistema de valores que la sociedad moderna promueve (10).

Desde esta perspectiva, situada más en la comprensión del fenómeno de las drogas en los jóvenes universitarios, como resultado de una dinámica social compleja, esta investigación reconoce cuatro aspectos claves para el análisis de las motivaciones en torno al consumo de estas sustancias en un grupo de jóvenes universitarios: el primero, la droga como un constructo social, producto de las contradicciones sociales; segundo, el joven universitario como sujeto sensible y pensante y coproductor de su realidad social; tercero, el consumo como una práctica recreativa y de ocio que transita entre la legitimidad e ilegitimidad, lo legal y lo ilegal; y, por último, la reflexión individual y colectiva como dispositivo necesario para un consumo responsable y consciente.

En cuanto a la droga como constructo social, es necesario reconocer que en cada cultura, desde antaño hombres y mujeres han descubierto los efectos de ciertas plantas para su beneficio, especialmente en el ámbito religioso y medicinal. El intercambio de conocimientos entre las distintas culturas de la Antigüedad en el contexto médico, llevó también al descubrimiento de ciertas propiedades placenteras y de alteración de los estados mentales. Esto último hizo que las drogas pasaran del terreno terapéutico al extramedicinal en el consumo popular y religioso.

En las sociedades modernas tuvo que diferenciarse el uso médico, del uso no médico de las drogas, estableciéndose regulaciones tanto legales como morales sobre su uso; sin embargo, de manera contradictoria, el comercio de las distintas sustancias psicoactivas benefició a las élites imperiales y mercantiles, que descubrieron que podían utilizar las drogas para controlar a trabajadores manuales y explotar a los indígenas. Así, el opio llevó a los trabajadores chinos a una situación de deuda y dependencia, en tanto que el alcohol obligó a los pueblos indígenas a comercializar sus pieles, vender a los cautivos como esclavos y hacerlos perder sus tierras (11).

En la actualidad, los regímenes normativos modernos dividen las drogas psicoactivas en distintas categorías como legales o ilegales, blandas o duras; no obstante, las drogas en general, legales o ilegales, son mercancías que se producen bajo la lógica y la racionali- 
dad capitalista: la producción, la distribución, la circulación y el consumo de un psicoactivo en las sociedades modernas no está por fuera de dicha racionalidad. Es por ello que la droga adquiere una connotación de constructo social, porque su representación es resultado del contexto histórico-cultural en el que se produzca y consuma (12).

Por ejemplo, tal como se mostró en los párrafos anteriores, en la sociedades precapitalistas los psicotrópicos fueron valores de uso producidos y utilizados para rituales religiosos y tratamientos médicos, en cambio, en las sociedades modernas actuales, en las que muchos de los valores morales están en función de la riqueza y la competitividad, las drogas son mercancías que permiten la acumulación de capital de quienes las producen y distribuyen y el deterioro de la vida de quienes las consumen, tal como lo muestran las estadísticas epidemiológicas presentadas al inicio de la introducción.

La segunda idea clave hace referencia al joven universitario como sujeto sensible y pensante, coproductor de su realidad. Para la literatura convencional, en el marco de la psicología evolutiva, la juventud se enmarca dentro del concepto de adulto joven, definido como una etapa en la vida de tránsito entre la adolescencia y la adultez, que se ubica entre los 18 y los 24 años de edad, con atributos personales como maduración en cuanto crecimiento y desarrollo psicológico y gran facilidad para el cambio, lo que facilita el aprendizaje y aprehensión de conocimientos e información a velocidad. Al finalizar el período, carreras terminadas, trabajo y relaciones de pareja con miras a adquirir responsabilidades de familia propia (13), pero para fines de esta investigación la juventud es entendida como un fenómeno sociológico que, en consecuencia, hay que entender desde la órbita de la reflexión sobre lo social humano y del devenir histórico y, por tanto, una construcción sociohistórica, cultural y relacional en las sociedades contemporáneas, es decir, una categoría social $(14,15)$.

Desde las corrientes críticas de la antropología, la sociología y la psicología social, se debate el concepto de juventud hacia la noción de juventudes, como manera de romper con la universalidad occidental. Por ello, se plantea que las juventudes son condiciones históricamente construidas y determinadas por diferentes variables que las atraviesan y que se podrían identificar con:

El sexo, que está determinado de manera
biológica; el género en el que se desarrolle
la interacción psíquica en los procesos de
socialización humana; la condición social
de hombre o de mujer que se haya asumido
para interactuar socialmente; la generación
o el ámbito temporal de construcción de la
experiencia individual y colectiva; la etnia
y, en general, las culturas contenidas en los
lenguajes con los que las sociedades aspiran
la comprensión interindividual; las oportu-
nidades socioeconómicas de las que logren
disponer las individualidades y las colectivi-
dades humanas, y las territorialidades, que
se constituyen en el espacio geográfico para
ser habitadas con los referentes. (14)

En este sentido, el joven universitario senti-pensante, coproductor de su realidad social, no es un sujeto único y universal, por el contrario, son sujetos diversos en cuanto a historias, formas de ver, sentir, apropiar y orientar sus experiencias vitales; actores protagónicos de su devenir histórico, y del devenir de la sociedad, que en el marco de las relaciones intersubjetivas en espacios de confianza, colectivos y reflexivos están en la capacidad y pueden optar por el diálogo crítico, reflexivo y constructivo, en función de encontrar nuevas maneras de comprender y transformar su realidad social. En el caso específico de las drogas, abiertos a entender 
como sujetos o no de consumo, movilizados por motivaciones internas y externas (16) y dispuestos al debate reflexivo, siempre y cuando los escenarios y espacios para ello no estén significados por prejuicios morales.

La tercera idea clave hace referencia al consumo de sustancias psicoactivas como una práctica recreativa y de ocio que transita entre la legalidad y la ilegalidad. A este respecto, lo primero que se debe resaltar es que las propiedades que tienen estas sustancias, en tanto que alteran los estados mentales y producen una sensación de placer psíquico en el organismo, han posibilitado su uso para fines religiosos y recreativos, y hoy en día también para fines económicos. Estas prácticas culturales no han sido iguales en todas las culturas y épocas - por eso su contenido histórico-, pero sí han estado presentes desde el inicio de la humanidad, y de ahí su carácter transhistórico (12).

Desde esta perspectiva, la comprensión de este fenómeno no se resuelve con una postura moral de lo correcto vs. lo incorrecto, lo bueno vs. lo malo, por el contrario, se requiere un análisis complejo, contextualizado y situado en los sujetos y en su momento histórico, para aproximarse a la naturaleza del fenómeno. En este sentido, es necesario precisar que el ocio es una necesidad humana para la cual se crean satisfactores relacionados con el ser, tener, hacer y estar, entre ellos la curiosidad, la imaginación, la despreocupación, divertirse, espacios de encuentro, entre otros (17), y en este marco de posibilidades, en todas las culturas los psicoactivos han sido utilizados como satisfactores. Lo problemático actualmente es que el consumo de psicotrópicos emerge de un contexto económico y político caracterizado por la mafia, la economía ilegal y la subsunción de la vida a los intereses del sistema capitalista.
Evidencia de ello son los informes presentados por la Organización de las Naciones Unidas contra la Droga y el Delito (UNODC, en inglés), los cuales muestran que a nivel mundial el tamaño total de los mercados de las drogas de uso ilícito puede llegar a unos \$320000 millones, o 0,9\% del PIB mundial, y en el caso del mercado de las drogas de uso legal, específicamente del tabaco, con las dos grandes multinacionales tabacaleras British American Tobacco (BAT) y Philip Morris International (PMI), se encontró que estas monopolizan el mercado de tabaco en América Latina. BAT, propiedad del grupo británico productor de los cigarros Kent, Dunhill, Lucky Strike y Pall Mall, canaliza el 60\% de sus utilidades en los países en vías de desarrollo, y Philip Morris International (PMI) se consolida como la empresa tabacalera transnacional que cotiza en la bolsa más rentable y más grande del mundo (12).

Estas cifras, tanto las generadas sobre el mercado de drogas de uso ilegal como aquellas acerca del mercado del cigarrillo como sustancia de uso lícito, muestran la alta rentabilidad económica que tienen estas sustancias en el sistema capitalista, situación que deja entrever la complejidad del fenómeno, en tanto que el ocio y uno de sus satisfactores ya no responden a una racionalidad que se sustenta en el esparcimiento y el crecimiento personal y espiritual, sino en ampliar un mercado para el consumo de drogas (legales o ilegales), en sujetos vaciados emocional y espiritualmente y contaminados fisicamente por una sociedad de consumo capitalista.

Por último, para la cuarta idea clave, que corresponde a la reflexión individual y colectiva como dispositivo necesario para un consumo responsable y consiente, se retoman los planteamientos de Humberto Maturana y Fritjof Capra con la categoría de autopoiesis, la cual definen como el patrón de organización de los organismos vivos, es decir, el "hacerse a 
sí mismo" en un patrón de red, en el que la función de cada componente es participar en la producción o transformación de otros componentes de la red, de tal modo que esta se produce a sí misma permanentemente. De esta manera, la entidad orgánica es producida por sus componentes y, a su vez, los produce (18).

Las sociedades humanas constituyen un caso especial, debido al papel crucial que desempeña en ellas el lenguaje, identificado por Maturana como el fenómeno crítico en el desarrollo de la conciencia y la cultura humana. Mientras que la cohesión de los insectos sociales se basa en el intercambio de compuestos químicos entre sus individuos, la unidad de las sociedades humanas se basa en el intercambio de lenguaje (18). Desde esta perspectiva, todo organismo vivo, incluyendo al ser humano, es un sistema autopoiético, es decir, tiene una estructura disipativa, en la cual el sistema vivo es a la vez abierto y cerrado: abierto estructuralmente, pero cerrado organizativamente. La materia y la energía fluyen a través de él, pero el sistema mantiene una forma estable, y lo hace de manera autónoma, a través de su autoorganización $(18,19)$.

Desde este marco interpretativo, el ser humano, como individuo y colectivo, se construye a sí mismo y construye sus experiencias de vida, a través de la intersubjetividad y del encuentro con otros, en procesos de socialización mediados por el lenguaje y las representaciones sociales. Este proceso es permanente, nunca se es un sujeto dado, con trayectorias de vida ya establecidas, somos sujetos historizados, construyéndonos siempre con el devenir histórico, somos sujetos autopoiéticos. En esta dinámica, lugares como la universidad y etapas como la vida universitaria, junto con la familia y demás escenarios donde se desenvuelve el individuo, son parte de ese entorno, de ese sistema vivo, con el que cada persona interactúa, del que recibe información y bienes materiales que él significa y transforma para sí de manera autónoma, en la construcción de su identidad y proyecto de vida.

Por ello, la universidad, como escenario académico para el debate, es un escenario ideal para el diálogo crítico y reflexivo en torno a la relación juventud-drogas, en donde los jóvenes están en la capacidad de construir sentidos individuales y colectivos, agrupando el presente y el pasado y orientando su futuro, mediante la percepción, la identificación y el reconocimiento mutuo y continuo de sus memorias colectivas y de sus posibilidades de respuesta, en la búsqueda de espacios de conciencia (20). Allí los jóvenes se pueden asumir como agentes sociales de transformación, a partir de sus propias realidades, en un marco institucional y relacional que la Universidad puede ofrecer de comprensión, equidad y respecto.

Todos los temas incluidos en esta introducción fueron en su momento, o premisas iniciales para la justificación de la investigación y su diseño metodológico, o reflexiones posteriores a los resultados, porque en ella se despliega una perspectiva más compleja e integradora que intenta dar cuenta del consumo de drogas en los jóvenes, como resultado de una red de determinaciones sociales, y no de decisiones aisladas, circunscritas a estilos de vida individuales.

El objetivo general de la investigación fue comprender los motivos que llevaban a un grupo de jóvenes universitarios de los programas académicos de Ciencias de la Salud y Pecuarias de la Universidad de Ciencias Aplicadas y Ambientales, al consumo de sustancias psicoactivas de uso legal e ilegal y, de manera más específica, se buscaba identificar relaciones entre situaciones del ámbito familiar y del ámbito universitario, al igual que circunstancias relacionadas con la relación entre pares y el rendimiento académico, con el inicio, consumo y abuso de sustancias psicoactivas. 


\section{Metodología}

\section{Tipo de estudio}

Esta investigación se planteó desde la perspectiva cualitativa, de estudio de caso, bajo un diseño fenomenológico, respondiendo a la siguiente pregunta: ¿Cuáles son los motivos que llevan a un grupo de jóvenes universitarios de los programas académicos de Ciencias de la Salud y Pecuarias de la Universidad de Ciencias Aplicadas y Ambientales al consumo de sustancias psicoactivas de uso legal e ilegal?

\section{Fuentes y técnicas de recolección de información}

Los sujetos participantes de la investigación fueron 32 jóvenes universitarios de los programas académicos de Medicina Humana, Enfermería, Zootecnia, Medicina Veterinaria y Medicina Veterinaria y Zootecnia, que actualmente estudian en la Universidad de Ciencias Aplicadas y Ambientales (UDCA). Se incluyeron personas de ambos géneros, de diferentes semestres, provenientes de distintos lugares del país, con prácticas religiosas y características personales diversas, todo ello con el fin de entender a los sujetos de consumo y no consumo, desde diversas experiencias de vida. Tres instrumentos sirvieron para recolectar la información: grupos focales, observación participante y observación no participante.

\section{Trabajo de campo}

Para este momento investigativo se llevaron a cabo tres actividades: la primera, una revisión sistemática de la literatura científica; la segunda, la realización de dos grupos focales por cada programa académico, con la participación de aproximadamente ocho integrantes por grupo. Para el desarrollo de esta actividad se diseñaron previamente algunas preguntas orientadoras que indagaban sobre el inicio, el tipo, las principales sustancias y los motivos de consumo, al igual que el rendimiento académico de los jóvenes que las consumían. También se dio vía libre a las preguntas y reflexiones relacionadas con el objeto de investigación, originadas en el interior de los grupos focales. Para la realización de los grupos focales se contó con un moderador a cargo de la dirección de la discusión, un observador-relator y grabadora de audio. La tercera actividad estuvo relacionada con la participación de la investigadora como observadora participante en dos actividades de ocio y recreación, tipo fiestas, y observación no participante en lugares de esparcimiento como bares y espacios deportivos.

\section{Análisis de la información}

Una vez se recolectó la información, se llevó a cabo la transcripción de las entrevistas grupales en un texto de Word y se elaboró una matriz para registrar allí la identificación de los momentos y las reducciones fenomenológicas por cada uno de los (las) jóvenes participantes, de acuerdo a los criterios establecidos por Leal (21) (tabla 1).

Por último, con el análisis de los datos obtenidos de las reducciones fenomenológicas y aplicando los principios de la codificación abierta, axial y selectiva, se identifican las categorías centrales y las subcategorías en distintos niveles (tabla 2).

\section{Consideraciones éticas}

Cumpliendo con los criterios éticos de la investigación en salud, se explicó y entregó el consentimiento informado, con capacidad de libre elección y sin coacción alguna, a los par- 
TABLa 1. Momentos Y ReducCIONES FENOMENOLÓGICAS

\begin{tabular}{|l|l|l|l|l|l|}
\hline Sujeto/persona & Dato & Psicológico & $\begin{array}{l}\text { Lógica del } \\
\text { significado }\end{array}$ & $\begin{array}{l}\text { Constitutivo trascen- } \\
\text { dental de cada sujeto }\end{array}$ & $\begin{array}{l}\text { Metafísico de la } \\
\text { conciencia }\end{array}$ \\
\hline & & & $\begin{array}{l}\text { (Aproximación a } \\
\text { quién es y qué piensa } \\
\text { el sujeto que habla en } \\
\text { cada relato, sobre las } \\
\text { sustancias psicoactivas) }\end{array}$ & $\begin{array}{l}\text { (A través de la com- } \\
\text { paración, quiénes son } \\
\text { los jóvenes universi- } \\
\text { tarios, diferencias y } \\
\text { divergencias) }\end{array}$ \\
\hline & & & & & \\
& & & &
\end{tabular}

Tabla 2. Categorías y SubCategorías deductivas e inductivas

\begin{tabular}{|c|c|c|c|}
\hline Categorías & $\begin{array}{c}\text { Subcategorías } \\
\text { primer nivel }\end{array}$ & $\begin{array}{l}\text { Subcategorías } \\
\text { segundo nivel }\end{array}$ & Subcategoría tercer nivel \\
\hline \multirow{8}{*}{$\begin{array}{l}\text { Consumo de } \\
\text { sustancias } \\
\text { psicoactivas } \\
\text { (CSP) }\end{array}$} & Tipo de sustancias (TS) & $\begin{array}{l}\text { Sustancias de uso legal } \\
\text { (sUL), sustancias de uso } \\
\text { ilegal (sUIL) }\end{array}$ & $\begin{array}{l}\text { Marihuana (Mh), alcohol } \\
\text { (A), tabaco (T), ácidos (A), } \\
\text { anfetaminas (Af) }\end{array}$ \\
\hline & Tipo de consumo (TC) & $\begin{array}{l}\text { Inicial - experimental (IE), } \\
\text { ocasional (O), frecuente } \\
\text { (F), dependiente (D), } \\
\text { Policonsumo (PC) }\end{array}$ & \\
\hline & Decisión personal (DP) & No consumo (NC) & \\
\hline & \multirow{4}{*}{ Motivaciones (M) } & Relación entre pares (REP) & $\begin{array}{l}\text { Aceptación y presión (AP), } \\
\text { encuentro afectivo (EA) }\end{array}$ \\
\hline & & Ámbito personal (AP) & $\begin{array}{l}\text { Problemas sentimentales } \\
\text { (Ps), baja autoestima (BA), } \\
\text { curiosidad (C), placer (P) }\end{array}$ \\
\hline & & Ámbito familiar (AF) & $\begin{array}{l}\text { Patrones familiares }(\mathrm{PF}) \text {, } \\
\text { Problemas familiares } \\
(\mathrm{PrF})\end{array}$ \\
\hline & & Ámbito académico (AA) & Exigencia académica (EA) \\
\hline & $\begin{array}{l}\text { Situaciones facilitadoras } \\
\text { del consumo (SFC) }\end{array}$ & $\begin{array}{l}\text { Oferta comercial (OC), } \\
\text { fácil acceso (FA) }\end{array}$ & \\
\hline \multirow{6}{*}{$\begin{array}{l}\text { Reflexiones } \\
\text { ético- morales } \\
\text { sobre el } \\
\text { consumo }\end{array}$} & Políticas de regulación (PR) & & \\
\hline & \multirow{2}{*}{ Tipo de sustancias (Ts) } & Uso legal e ilegal (ULI) & \\
\hline & & Lugares y momentos (LM) & Espacios de libertad (EL) \\
\hline & Finalidad del consumo (FC) & & \\
\hline & \multirow{2}{*}{ Sujeto de consumo (sc) } & Autocontrol (Act) & \\
\hline & & & Resistencia social (RS) \\
\hline
\end{tabular}

Fuente: proceso de codificación y categorización de la investigación 
JUVENTUD Y UNIVERSIDAD: SUJETOS Y ESCENARIOS PARA EL DEBATE CRITICO Y AUTORREFLEXIVO SOBRE EL CONSUMO DE SUSTANCIAS PSICOACTIVAS DE USO LEGAL E ILEGAL

ticipantes al inicio de los grupos focales, con base en la Resolución №. 008430 de 1993 (22).

\section{Resultados y discusión}

La investigación arroja dos categorías centrales que expresan la experiencia de los (las) jóvenes universitarios con respecto a las sustancias psicoactivas, la primera de ellas relacionada con el consumo y la segunda con la reflexión ético-moral del consumo. Cada una se despliega en un entramado de relaciones entre subcategorías de primer, segundo y tercer nivel de jerarquía, dependiendo de la relación que se estableció, de pertenencia, propiedad, determinación, atributo o manifestación. En la figura 1 se muestra que la experiencia de consumo de sustancias psicoactivas, o por el contrario, el no consumo de estas sustancias, es resultado de la decisión personal, pero asimismo, el consumo se constituye en un determinante para decidirse por el no consumo, o el consumo autocontralado, como consecuencia de experiencias negativas propias o ajenas, o por autorreflexión (figura 2). En los relatos, los jóvenes lo expresan así:

Ya es tu decisión si lo haces, porque yo puedo andar con mis amigas que les gusta, pero si yo digo que no, entonces es algo ya muy personal. (M5GF2CSE)

$[\ldots]$ no consumo $[\ldots]$ por miedo a ver a personas muy perdidas en eso. (H3GF2CPZMV)

[...] incluso muchos ni siquiera quieren llegar al grado de estar prendidos, no sé, con una cerveza o medio cerveza ya estuvo y ya están disfrutando exactamente sin estar tomando. (M1GF2CSM)

Es mi experiencia que todos tenemos un punto y todos sabemos hasta donde llegar y si me ha pasado a mí, yo digo, yo ya no fumo más porque ya no me siento bien, porque ya siento que voy a embarrarla pues no fumo más. (M1GF2CSE)

Como práctica, el uso de sustancias psicoactivas en los jóvenes entrevistados se manifiesta en el tipo de sustancia consumida y los tipos de consumo. En cuanto a las sustancias, todos los grupos concordaron en que la sustancia de uso legal más consumida es el alcohol, después el tabaco, y de uso ilegal la marihuana. Los ácidos, la cocaína y las anfetaminas fueron referenciados, pero con menor frecuencia. Estos datos coinciden con el Informe Mundial sobre Drogas de los años 2012, 2013 y 2014, de la UNODC (1-3), con los reportes del Gobierno Nacional de la República de Colombia, en el Estudio Nacional de Consumo de Sustancias Psicoactivas del 2013 (4), y con el estudio de la Secretaría General de la Comunidad Andina, II Estudio Epidemiológico Andino sobre Consumo de Drogas en la Población Universitaria, Informe Regional de 2012 (5).

De los tipos de consumo, los estudiantes hablaron de consumo inicial-experimental, ocasional, frecuente, dependiente y policonsumo (figura 1):

Cada ocho días en la 170, cada ocho días tomando. (H2GF1CSE)

Lo que pasa es que la mayoría de actividades grupales de las universidades, pues extracurriculares, cuando salen entonces, pues generalmente es a bares, a bailar, a sentarse a hablar, a mirar partidos, entonces pues. Cuando uno va a esos sitios, hay que, pues hay que consumir alcohol, porque uno entra a los sitios y entra pues a consumir alcohol. (M3GF2CSM)

En cuanto a la decisión personal, se observa en el análisis de los datos que esta se comporta como un elemento catalizador entre 


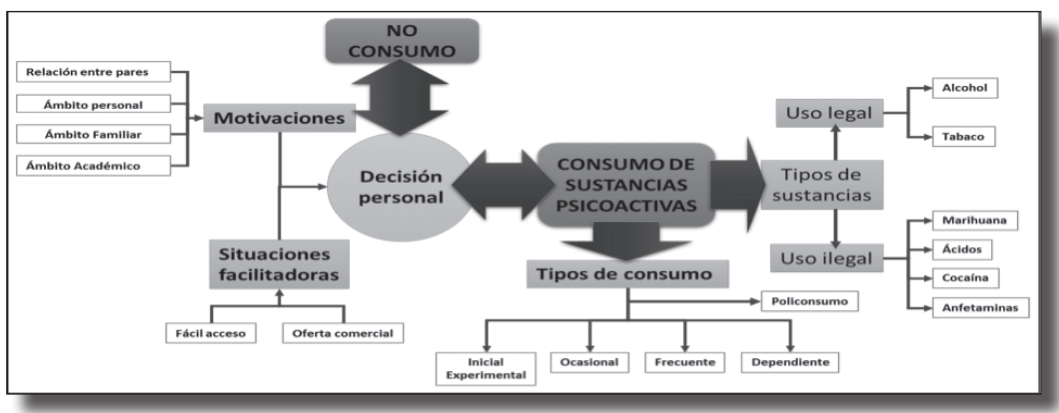

Figura 1

Fuente: proceso de categorización axial y selectiva

las motivaciones, las situaciones facilitadoras y el consumo o no consumo de sustancias psicoactivas de los jóvenes participantes (figura 1). Esto en razón a su alta densidad en los relatos, reflejada en el debate de todos los grupos focales, por varios de sus participantes, como la instancia última en la que el (la) joven opta por consumir, no consumir o tener un consumo controlado una vez haya estado movilizado por motivaciones internas del ámbito personal, externas del ámbito familiar, académico, de relación entre pares (figura 2) o expuesto a situaciones facilitadoras del consumo (figura 1). Este hallazgo se evidencia en los siguientes relatos:

[...] por lo que los demás dicen y todo el mundo lo hace y [...] iuy! si yo no lo hago estoy fuera y esa gente me va a dejar de hablar y voy a estar solo, decir no, no lo hago y de malas al que no le guste pues [...] (H2GF2CSM)

[...] si usted no quiere seguir fumando se siente mal haciéndolo pues entonces no fume, pero no van a estar ahí, no van a estar ahí las personas diciéndole, fume usted tiene que fumar, es que si usted no fuma...pues no. (M1GF2CSE)

$\mathrm{Al}$ comparar este hallazgo con otras investigaciones, se encuentra que la decisión personal surge como factor asociado al consumo, tal como lo muestra la investigación de Varela et al. sobre consumo de sustancias psicoactivas ilegales en jóvenes en la ciudad de Cali (23), el estudio realizado en México por Rodríguez y su equipo de investigación sobre evaluación de un programa de prevención del consumo de drogas para ado-

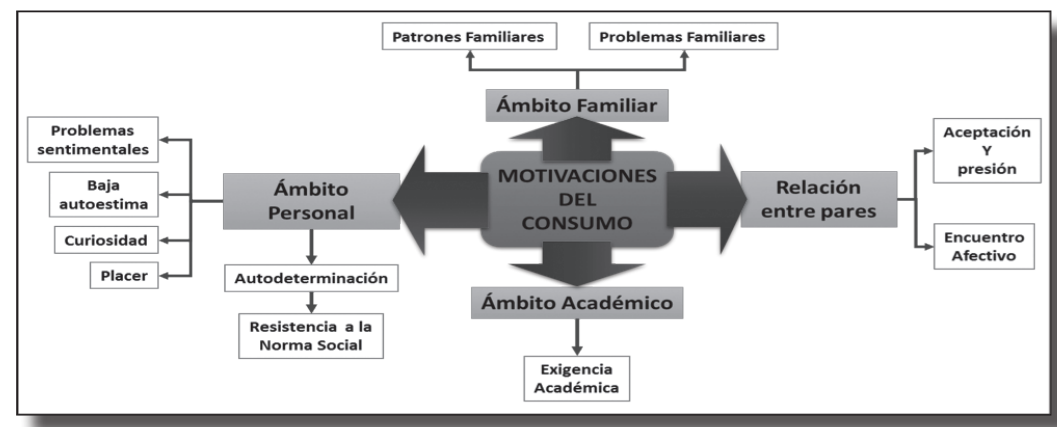


JUVENTUD Y UNIVERSIDAD: SUJETOS Y ESCENARIOS PARA EL DEBATE CRITICO Y AUTORREFLEXIVO SOBRE EL CONSUMO DE SUSTANCIAS PSICOACTIVAS DE USO LEGAL E ILEGAL

lescentes (24), así como el trabajo de López Torrecillas sobre autocontrol y consumo de drogas en España (25). Sin embargo, en esta investigación, el estatus responde a un determinante significativo en la frontera entre el consumo controlado, el abuso en el consumo o el no consumo, que no esta tan claramente definido en las anteriores investigaciones.

El énfasis en este aspecto puede marcar la diferencia en la dirección que tengan los diferentes programas de prevención de consumo de sustancias psicoactivas, con respecto a continuar una política prohibicionista y punitiva, o por el contrario, reconocer a los jóvenes como actores claves en el diseño de estrategias que promuevan espacios de diálogo, el debate crítico y la toma de decisiones asertivas sobre el consumo de psicoactivos.

Por su parte, las subcategorías motivaciones y situaciones facilitadoras del consumo emergen en el estudio como determinantes del consumo, desde la percepción y vivencia del grupo de jóvenes participantes. Las motivaciones provienen de tres ámbitos: el académico, el personal y el familiar, y también de la relación entre pares. Del ámbito académico, la exigencia, específicamente durante la época de parciales: “...también eso, por el estrés yo creo [...] digamos uno termina un parcial y uno como que me fue bien o me fue mal, entonces bueno, vamos a tomar o vamos a consumir algo" (H1GF2CPMVZ).

Del ámbito personal se identificaron varias motivaciones que promueven el consumo en estos jóvenes: problemas sentimentales, baja autoestima, curiosidad, placer y autodeterminación (figura 2). La curiosidad es la motivación para el inicio del consumo de cualquier sustancia, con la mayor densidad en los relatos:

[...] yo creo que la curiosidad (H1GF2CPZMV).
[...] si comparto su idea...pero es que yo creo que es la principal [...] (H2GF2CPZMV)

pues yo digo que curiosidad porque es lo que encierra todo, ino? (H3GF2CPZMV)

cuando yo probé la marihuana por primera vez fue curiosidad y si quería saber que se sentía y después me llamo mucho la atención. (M1GF2CSE)

Digamos uno llega a primero y en mi salón por ejemplo, había gente que, ehh... pues yo fumo, entonces uno salía a fumar y se le pegaba otra persona no déjame probar y empiezan ahí con eso. (M2GF2CSM)

Este último hallazgo, como un determinante importante para el inicio del consumo de sustancias psicoactivas, está muy bien documentado en el campo de la ciencias de la salud y en las ciencias humanas. Así lo demuestra la revisión realizada por Rodríguez Suárez et al (26).

Con respecto a la autodeterminación, que tiene una relación empírica y teórica con la categoría decisión personal, si bien no tiene una densidad tan alta en el estudio, se considera relevante el hallazgo, por cuanto marca una diferencia con otros trabajos que explican el fenómeno del consumo de drogas, o del no consumo, desde los factores de riesgo o protectores, sin profundizar en cómo se construye una actitud autodeterminada, como proceso protector y no como un simple factor que suma a la red de causalidades.

La autodeterminación para los participantes hace referencia a la actitud consciente del consumidor o no consumidor, más allá de estándares que la sociedad impone como normas sociales a los individuos. No es el miedo, ni el cumplimiento de la norma por la norma, o la actitud obediente ante la autoridad, o por el contrario, la actitud 
desobediente sin sentido, lo que controla o promueve el consumo, sino la decisión meditada, dialogada y teleológica del consumo o no consumo, lo que determina el actuar de la persona (figura 3 ).

En varios momentos del debate en los grupos focales, algunos participantes hacían referencia al consumo consciente como un dispositivo para el autocontrol y facilitador de escenarios para el crecimiento personal y espiritual, situación que se acerca a las prácticas ancestrales de las comunidades indígenas de América y de las sociedades antiguas en todo el planeta (11). A continuación se presentan algunos relatos que desde diferentes ángulos hablan del consumo o no consumo consciente:

La orquesta " $X$ " antes de tocar tiene que meterse su porriada o sino no pueden tocar... son músicos muy buenos y como personas son excelentes, pero pasa y es raro, porque si se supone que es una sustancias psicoactiva tiene que generar cosas malas, pero ahí no las genera, yo no estoy de acuerdo igual, porque es algo malo, tanto para la persona como para la sociedad, pero sí en esos casos y cuando una persona es controlada y sabe lo que está haciendo nunca he visto cosas que lleguen a extremos malos. (H2GF2CSM)

Cuando nosotros fumamos es una especie de liberación porque realmente yo lo siento así, y yo me libero... no tengo que estar pensando en otras cosas que realmente me roban esa felicidad interna que uno realmente siente...a uno le están robando pedacitos porque le está robando parte de su ser [...] el consumo va más allá... me sentía como el ser que en realidad soy yo [...] o sea cuando uno pierde el sentido de porque está consumiendo, créalas que va a terminar por allá en la L si le va mejor. (M1GF2CSE)

Yo pienso que ahí es cuestión de cómo crecimiento personal, me entiendes, si yo sé y siento que estoy preparado para probarlo, pues lo hago, ipero si no? si yo en este momento digo no, no estoy preparado, pues no lo hago. (M4GF2CSE)

Las narraciones anteriores recogen la participación de dos jovenes consumidores y un no consumidor de marihuana, dos aceptan el consumo cuando este es resultado de una actitud autocontrolada y otro cuando la finalidad es el encuentro consigo mismo. Se resalta de este hallazgo la actitud crítica de los estudiantes, como un dispositivo desde el cual pueden emerger otros abordajes preventivos en relación con el consumo, más situados en el sujeto, en su capacidad de decisión y discernimiento y no desde las medidas prohibicionistas que reclaman el "deber ser" y niegan el fenomeno en su real dimensión. Hoy en día existen perspectivas alternativas que exploran abordajes en una relación horizontal con los jóvenes, en las que se cuestionan los estereotipos sociales creados acerca de estos como personas conflictivas. Tal es la propuesta de Pacheco y Martínez (27), quienes se preguntan qué hace efectiva una intervención en el consumo de drogas, a lo que responden que es el sistema de creencias de los terapeutas, en cuanto a creer en el cambio a través del programa, creer en el adolescente usuario y creer en ellos mismos como promotores de ayuda para el cambio, además de la opinión consensuada sobre la adolescencia como etapa de la vida que se aleja de estereotipos negativos, lo que garantiza un mayor éxito en los tratamientos de rehabilitación.

En cuanto a las motivaciones del ámbito familiar, la investigación reporta que los problemas y patrones familiares de consumo también son percibidos por los participantes como condiciones que favorecen el inicio del consumo en todas sus tipologías y de todo tipo de sustancias (figura 2), hallazgos coincidentes con muchas de las investigaciones 
realizadas en este campo, como la de Veloza et al., sobre medio social y uso simultáneo de alcohol y tabaco en estudiantes universitarios de pregrado de carreras de ciencias de la salud de una universidad (28); la de López, Santín et al., acerca del consumo de sustancias psicoactivas en una muestra de jóvenes universitarios en Veracruz, México (29); la de Duffy, con respecto a factores de riesgo y factores protectores asociados al consumo de alcohol en niños y adolescentes (30); así como la de Mathews, que indaga sobre los factores protectores y de riesgo asociados al uso de alcohol en adolescentes hijos de padre alcohólico en el Perú (31). Todas ellas muestran que los patrones familiares de consumo y los problemas familiares son determinantes en el consumo de los jóvenes.

La relación entre pares, mencionada por los (as) jóvenes como otro ámbito en el que surgen motivaciones para el consumo, es otro de los determinantes con alta densidad en el estudio, bastante investigado en este campo. Allí se identifican dos condicionantes: el primero, la necesidad de aceptación en el grupo de amigos(as), compañeros (as), que puede estar acompañada de la presión que estos(as) ejercen para iniciarse o mantenerse en el consumo; y el segundo, la necesidad de encuentro afectivo con los(as) amigos (as) (figura 2). Este último hallazgo es muy importante para algunos de los participantes, no en términos del joven sujeto de consumo carente de afecto, sino como el sujeto de consumo, motivado y movilizado por el sentir y la necesidad de construir relaciones afectivas con sus pares. El consumo, no mirado exclusivamente como una huida de la angustia fundamental de la existencia, como lo plantea la psicología convencional, sino como una actividad que le posibilita al sujeto entrar en un mundo de relaciones sociales cargadas de significación, mundo en el que asienta su existencia, tal como lo argumenta Sierra en su trabajo investigativo sobre drogas y universidad, llevado a cabo en la ciudad de Quito (11).

Por su parte, las situaciones facilitadoras, junto con las motivaciones, constituyen el segundo determinante que, de acuerdo a los resultado de este estudio, favorece el inicio y mantenimiento del consumo de sustancias psicoactivas. A esta situación responde el fácil acceso a la sustancias y la amplia oferta comercial para el consumo de alcohol y tabaco, así como la oferta de sustancias de uso ilegal. Entre los relatos que resultaron significativos están los siguientes:

\section{[...] es la facilidad de conseguir ahora todo, o sea, antes era muy complicado [...] ahorita ya no, o sea, ya es muy común entrar a un bar y que te pregunten bueno vas a consumir, no sé, pepas o tal cosa, o vas a consumir licor que vas a hacer. (M1GF2CSM)}

O sea uno va a un bar y si no compra entonces no lo dejan entrar o si no consume no tal cosa. (M4GF2CSM)

Y los amigos si tienen plata para prestarle a uno cuando va a tomar, pero uno le dice préstame para almorzar: no, no tengo... pero pa' tomar: ¿cuánto necesitas? (M2GF2CSM)

El problema del microtráfico y expendio de alcohol a los jóvenes y adolescentes en lugares cercanos al colegio y a las universidades es un asunto crítico, investigaciones como las de Rodríguez Suárez et al. (24), Varela Arévalo et al. (23) y Amador Calafat Far, director de la Revista de Adicciones (32), muestran, al igual que en esta investigación, que este es un determinante importante para el inicio y consumo regular de sustancias psicoactivas por parte de esta población.

Por último, la tercera categoría central en esta investigación, que recoge la percepción y vivencia de los jóvenes participantes acerca 
del consumo de sustancias psicoactivas, es la capacidad de reflexión ético-moral sobre esta práctica. Este hallazgo es muy relevante, porque sitúa la discusión sobre el consumo de drogas, no solo en el plano académico-científico, sino también en el plano ético-político, pues tal como lo plantean los jóvenes, el problema y el foco de atención no deben ser las sustancias, sino los sujetos de consumo, de no consumo y los sujetos auto controlados(as), aspecto que se logró vivenciar en los grupos focales, no exclusivamente como instrumentos para la recolección de información, sino también como espacios para la reflexión y el debate colectivo.

A este respecto los hallazgos muestran lo siguiente: cuando se genera un espacio de debate en torno al tema de consumo, los jóvenes transcienden el relato anecdótico y dejan ver su postura ética, moral y política sobre al tema. Uno de ellos se expresó así del grupo focal:

\footnotetext{
Por ejemplo, esto es un espacio bacano, bonito, siento buena energía acá, severo. Pero hay... hay gente, hay gente que no la siente, isi me hago entender? Son, son... cuando uno se moviliza por cosas así sencillas de compartir, de discutir, de...y creando, creando conocimiento y aprendizaje acá, uno no necesita estar en un salón para aprender. (H1GF2CSE)
}

Las reflexiones de los estudiantes trataron temas como las políticas de regulación prohibicionistas, con las que algunos no estaban de acuerdo, pero otros sí. También hablaron del daño que ocasionan las sustancias sobre el organismo y los niveles de dependencia. Cuestionaron asimismo la doble moral de la sociedad, al aceptar el tabaco y el alcohol y sancionar la marihuana, cuando las tres sustancias, en el especial las dos primeras, traen consecuencias nocivas para la persona y, sin embargo, son aceptadas por su carácter de legalidad. Algunos recalcaron la importancia de diferenciar los momentos y espacios de consumo, de aquellos donde la finalidad es estudiar o compartir otro tipo de actividades de ocio.

En varios grupos focales se priorizó la necesidad de centrar el abordaje del consumo, no desde las sustancias, sino desde el sujeto, pues es este quien decide en últimas qué hacer, si mantener un consumo controlado y consciente, no consumir, o dejar que las circunstancias y las sensaciones "decidan por él o ella”. El autocontrol y la decisión personal fueron dos temas de bastante debate y reflexión. Para algunos, el autocontrol proviene de la autorreflexión y poner límites propios; otros, en cambio, planteaban que el autocontrol proviene del respeto a las otras personas y a los espacios, en tanto que algunos afirmaban que el control se construye por las bases morales construidas en la familia (figura 3).

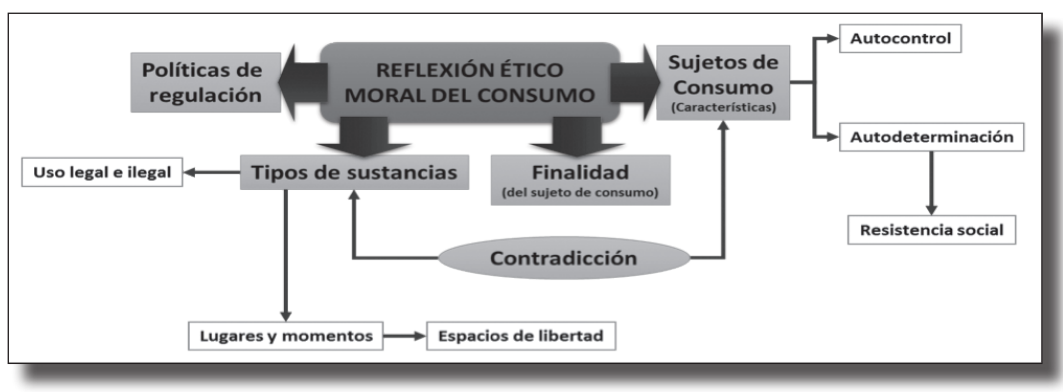

Figura 3

Fuente: proceso de categorización axial y selectiva 
En últimas, la riqueza de la investigación en este aspecto estuvo dada por el espacio de reflexión que se generó en torno al tema, el cual se convierte en sí mismo en un hallazgo relevante, y lo segundo por la diversidad de opiniones y argumentos sobre la valoración ética, moral y política del consumo de drogas por parte de los jóvenes, lo que revela un panorama más completo de su sentir y su pensar.

La mayoría de los estudios centran su atención en la descripción del fenómeno en términos de tipo de sustancia consumida, la frecuencia, la distribución en tiempo y espacio, la predicción de factores de riesgo y protectores, en tanto que algunos logran profundizar sobre en el autocontrol y la autoeficacia como atributos del sujeto que mantiene un consumo controlado, pero pocos exploran los espacios colectivos de debate y reflexión crítica como un escenario en el que el joven, además de presentar y argumentar el tema de la drogas desde su vivencia y percepción, construya habilidades sociales para establecer una relación responsable y consciente con las sustancias psicoactivas y adopte una postura crítica en relación con este fenómeno. Aunque esta investigación no tiene el alcance de evaluar este tipo de estrategias, como medidas preventivas del consumo abusivo, o promocionales en cuanto a una autonomía del sujeto en relación con las drogas, sí pone al descubierto la capacidad de los jóvenes para propiciar un debate argumentado al respecto, que en un trabajo más sostenido y creativo servirá como estrategia de intervención social sobre esta problemática.

\section{Conclusiones}

El consumo de sustancias psicoactivas es una práctica social e histórica, presente en todas las culturas y épocas de la humanidad como satisfactor de las necesidades de ocio y de transcendencia; sin embargo, para las sociedades capitalistas modernas occidentalizadas, esta práctica ha representado un deterioro importante en la red de relaciones sociales, por el tema específico de narcotráfico y la violencia, y en el caso de los jóvenes con consumo problemático y drogodependencia, el deterioro de su vida, como lo muestran estadísticas de los últimos años, obtenidas de la vigilancia epidemiológica, como evento de interés en salud pública.

Si bien esta investigación tiene limitaciones en cuanto a que su diseño metodológico no permite generalizar los resultados hacia todo el universo del que hace parte, el grupo de jóvenes que participaron del estudio de caso, también es cierto que a partir del horizonte de realidad expuesto anteriormente, sus resultados muestran hallazgos muy relevantes desde su percepción y vivencia, que podrían servir en el futuro como base e insumo para la problematización del consumo de drogas en este tipo de población.

Para los participantes, cuatro aspectos resultaron concluyentes al analizar los datos: el primero, ratificar la necesidad de diferenciar los tipos de consumo, inicial-experimental, ocasional, frecuente y dependiente, así como el no consumo, el consumo autocontrolado y el consumo consciente, pues ello aporta a una compresión más real y no totalizante del sentido de esta práctica para estos jóvenes universitarios. Segundo, entender que para estas personas, el consumo de drogas es una realidad que transciende el plano individual y se ubica como un fenómeno complejo, producto de una red de determinaciones que se cristalizan en el individuo, en sus decisiones personales y sus motivaciones, pero conectadas y movilizadas por sus experiencias en otros ámbitos como el de la familia, el académico y el mercado legal e ilegal de sustancias psicoactivas. 
El tercer hallazgo relevante hace referencia a las decisiones personales, como un catalizador social entre las determinaciones del entorno socio-cultural y aquellas situadas en la autonomía, las preferencias y motivaciones del individuo. El estatus empírico que adquiere esta categoría en el estudio ofrece una alternativa en cuanto a la necesidad de promover estrategias de intervención situadas en el fortalecimiento de la autonomía y la capacidad de toma de decisión por parte del sujeto, más que en medidas prohibicionistas, punitivas y moralistas.

Cuarto, las reflexiones centradas en el sujeto, no como un problema, sino como un sujeto histórico, contextualizado y capaz de tomar decisiones, no necesariamente asertivas en todos los casos, ubican el tema del consumo de drogas en un lugar más potenciable; podría situarse en la persona, en su sentir-pensar y actuar, de tal manera que las estrategias preventivas y promocionales actúen como dispositivos para cuidar la vida, y no solo para mantener un statu quo social, cada vez más cuestionado por la juventud.

Por último, la universidad, como escenario propicio para el debate crítico y reflexivo en torno al tráfico y consumo de sustancias psicoactivas, con la participación activa de los jóvenes, en un diálogo horizontal, puede abrir vías de transformación de esta problemática.

\section{Referencias bibliográficas}

1. United Nations Office and Drugs and Crime (UNODC). Informe Mundial sobre las Drogas. New York, 2012 [Internet] [acceso: 8 de septiembre del 2014]. Disponible en https://www.unodc.org/ documents/dataandanalysis/WDR2012/Executive_summary_spanish.pdf.

2. United Nations Office and Drugs and Crime (UNODC). Informe Mundial sobre las Drogas. New York, 2014 [Internet] [acceso: 8 de septiembre del 2014]. Disponible en: http://www.unodc.org/documents/ lpobrazil//noticias/2014/06/exec_summary_ES.pdf.
3. United Nations Office and Drugs and Crime (UNODC). Informe Mundial sobre las Drogas [Internet]. New York, 2013 [acceso: 8 de septiembre del 2014]. Disponible en http://www.unodc.org/documents/ lpobrazil//Topics_drugs/WDR/2013/Executive Summary_translation_-_Spanish_-_V1383706.pdf.

4. Gobierno Nacional de la República de Colombia. Estudio Nacional de Consumo de Sustancias Psicoactivas en Colombia 2013. Bogotá, 2014 [Internet] [acceso: 12 de septiembre del 2014]. Disponible en: https://www.unodc.org/documents/colombia/2014/ Julio/Estudio_de_Consumo_unodc.pdf.

5. Secretaría General de la Comunidad Andina. II Estudio Epidemiológico Andino sobre Consumo de Drogas en la Población Universitaria. Informe Regional, 2012. Perú, 2013 [Internet] [acceso: 14 de septiembre del 2014]. Disponible en: http://www. cicad.oas.org/oid/pubs/PRADICAN_Informe_Regional.pdf.

6. Morales B, Plazas M, Sánchez R, Ventura C. Factores de riesgo y de protección relacionados con el consumo de sustancias psicoactivas en estudiantes de enfermería. Rev Latino-Am. Enfermagem. 2011; 19 (número especial): 673-83. [Internet] [acceso: 15 de octubre del 2014]. Disponible en: http://www.scielo.br/scielo.php?pid=S0104$11692011000700003 \&$ script $=$ sci_arttext.

7. Cáceres D, Salazar I, Varela M, Tovar J. Consumo de drogas en jóvenes universitarios y su relación de riesgo y protección con los factores psicosociales. Univ Psychol. 2006; 5 (3): 521-34 [Internet] [acceso: 14 de septiembre del 2014]. Disponible en: http://www. scielo.org.co/pdf/rups/v5n3/v5n3a08.pdf.

8. Castañeda G. Sentidos del consumo de sustancias psicoactivas en la vivencia universitaria. Federación Internacional de Universidades católicas (FIUC), 2012 [Internet] [acceso: 20 de octubre del 2014]. Disponible en: http://virtual.funlam.edu.co/repositorio/sites/default/files/repositorioarchivos/2012/03/ articulo\%20revista\%20analisis.1369.pdf.

9. Prieto $\mathrm{R}$ et al. Diversidad y complejidad en el fenómeno de las drogas: el policonsumo simultáneo en estudiantes universitarios en una universidad, Cundinamarca - Colombia. Texto Contexto - Enfermería. 2012; 21: 49-55. [Internet] [acceso: 20 de junio del 2013]. Disponible en: http://www.scielo. br/scielo.php?script $=$ sci arttext $\&$ pid $=$ S01040707 2012000500006\&lng $=$ en $^{\prime}$ nrm $=$ iso.

10. Jáuregui I. Drogas y sociedad: la personalidad adictiva de nuestro tiempo. Nómadas. Revista Crítica de Ciencias Sociales y Jurídicas. 2007; 16 [Internet] [acceso: 15 de noviembre del 2014]. Disponible en: http://pendientedemigracion.ucm. es/info/nomadas/16/ijbalenciaga.pdf.

11. Sierra N. Drogas y universidad. Consejo Nacional de Control de Sustancias Estupefacientes y Psicotrópicas (Consep). Quito, 2012 [Internet] [acceso: 15 de noviembre del 2014]. Disponible en: http:// www.consep.gob.ec/descargas/2014/05/drogas-yuniversidad.pdf.

12. Cáliz N. La reproducción social de la vida en disputa con la reproducción del capital. Documento de trabajo de tesis doctoral. Bogotá, Colombia, 2014. 
13. Mansilla M. Etapas del desarrollo humano. Revista de Investigación en Psicología. 2000; 3 (2) [Internet] [acceso: 26 de noviembre del 2014]. Disponible en: http://revistasinvestigacion.unmsm.edu.pe/index. $\mathrm{php} / \mathrm{psico} / \mathrm{article} / \mathrm{view} / 4999$.

14. Villa M. Del concepto de juventud al de juventudes y al de lo juvenil. Revista Educación y Pedagogía. 2011; 23 (60) [Internet] [acceso: 22 de noviembre del 2014]. Disponible: http://aprendeenlinea. udea.edu.co/revistas/index.php/revistaeyp/article/ viewArticle/11421.

15. Feixa C. Generación XX. Teorías sobre la juventud en la era contemporánea. Revista Latinoamericana de Ciencias Sociales, Niñez y Juventud. 2006 JulDic; 4 [Internet] [acceso: 18 de marzo del 2015]. Disponible en:<http://www.redalyc.org/articulo. oa? id $=77340202>$ ISSN $1692-715 X$.

16. Hernández V, Scherer A. Motivação hacer estudante universitário para o consumo de drogas legais. Rev Latino-Am Enfermagem. 2008; 16 (número especial): 572-576 [Internet] [acceso: 18 de marzo del 2015. Disponible en: $<$ http://www. scielo.br/scielo.php?script $=$ sci arttext\&pid $=$ S0104$11692008000700011 \& \operatorname{lng}=\mathrm{en} \& \mathrm{nrm}=\mathrm{iso}>$.

17. Max Neef M. Desarrollo a escala humana: Conceptos, aplicaciones y algunas reflexiones. Montevideo: Nordan-Comunidad; 1998 [Internet]. Disponible en: http://www.max-neef.cl/descargas/Max_NeefDesarrollo_a_escala_humana.pdf.

18. Capra F. La trama de la vida: Una perspectiva de los sistemas vivos. Barcelona: Anagrama; 1998 [Internet]. Disponible en: http://cdm2011b.aprenderapensar.net/ files/2011/07/Capra_Fritjof_La_trama_de_la_vida.pdf.

19. Maturana H. El sentido de lo humano. Santiago de Chile: Ediciones Pedagógicas Chilenas; 1992 [Internet] [acceso: 26 de noviembre del 2014]. Disponible en: http://www.iutep.tec.ve/uptp/images/Descargas/ materialwr/libros/HumbertoMaturana-ElSentidodeloHumano.pdf.

20. Estrada J, Escobar D, Briceño J. Educación de pares como estrategia de prevención de la infección por el VIH en adolecentes: referentes teóricos y metodológicos. Revista Gerencia y Políticas de Salud. 2011; 10 (20): 46-66 [Internet] [acceso: 22 de noviembre del 2014]. Disponible en: http://www.scielo.org.co/scielo.php?pid=S165770272011000100004\&script=sci_arttext.

21. Leal N. El método fenomenológico: Principios, momentos y reducciones [Internet] [acceso: $27 \mathrm{de}$ noviembre del 2014]. Disponible en: http://revistadip. una.edu.ve/volumen1/epistemologia1/lealnestorepistemologia.pdf.

22. Ministerio de Salud. República de Colombia. Resolución $\mathrm{N}^{\circ} .008430$ de 1993 [Internet] [acceso: 16 de octubre del 2014]. Disponible en: http://www. unisabana.edu.co/fileadmin/Documentos/Investigacion/comite de etica/Res 84301993 - Salud.pdf.

23. Varela M, Salazar I, Cáceres D, Tovar J. Consumo de sustancias psicoactivas ilegales en jóvenes: factores psicosociales asociados. Pensamiento Psicológico.
2007; 3 (8): 31-45 [Internet] [acceso: 26 de noviembre del 2014]. Disponible en:http://revistas.javerianacali. edu.co/index.php/pensamientopsicologico/article/ view/70/208.

24. Rodríguez S, Bruno D, Gracia S, Guerrero J, GómezMaqueo E. Evaluación de un programa de prevención del consumo de drogas para adolescentes. Salud Mental. 2011; 34 (1), 27-35 [Internet] [acceso: 26 de noviembre del 2014]. Disponible en: http://www. scielo.org.mx/pdf/sm/v34n1/v34n1a4.pdf.

25. López-Torrecillas F, Peralta I, Muñoz-Rivas M, Godoy J. Autocontrol y consumo de drogas. Adicciones. 2003; 15 (2): 127-36 [Internet] [acceso: 27 de noviembre del 2014]. Disponible en: http://www.adicciones.es/ files/03-Autocontrol\%20y\%20consumo.pdf.

26. Rodríguez J, Agulló E, Agulló M. Jóvenes, fin de semana y uso recreativo de drogas: evolución y tendencias del ocio juvenil. Adicciones. 2003; 15 (Supl. 2) [Internet] [acceso: 18 de marzo del 2015]. Disponible: http://www.adicciones.es/revista new. php?register_vars[nume] $=61$.

27. Pacheco A, Martínez A. El arte de ser terapeuta ¿Qué hace efectiva una intervención en el consumo de drogas? Enseñanza e Investigación en Psicología. 2013; 18 (1): $159-76$ [Internet] [acceso: 16 de noviembre del 2014]. Disponible en: http://www.redalyc.org/articulo. oa?id=29228948011.

28. Veloza M, Simich L, Strike C, Brands B, Giesbrecht N, Khenti A. Medio social y uso simultáneo de alcohol y tabaco en estudiantes universitarios de pregrado de carreras de ciencias de la salud de una universidad, Cundinamarca - Colombia. Texto Contexto - Enferm. 2012; 21 (número especial): 41-48 [Internet] [acceso: 22 de noviembre del 2014. Disponible en: http:/www.scielo.br/scielo.php?pid=S010407072012000500005\&script=sci_arttext.

29. López M, Santín C, Torrico E, Rodríguez J. Consumo de sustancias-psicoactivas en una muestra de jóvenes universitarios. Psicol Salud. 2003 Ene-Jun; 13 (1): 5-18 [Internet] [acceso: 26 de noviembre del 2014]. Disponible en: http://revistas.uv.mx/index. php/psicysalud/article/viewFile/934/1723.

30. Duffy, D. Factores de riesgo y factores protectores asociados al consumo de alcohol en niños y adolescentes. Salud \& Sociedad. 2014: 5 (1): 40-52 [Internet] [acceso: 18 de marzo del 2015]. Disponible en: $<$ http://pepsic.bvsalud.org/scielo.php?script=sci arttext\&pid $=$ S0718-74752014000100003\&lng $=$ pt\& $\mathrm{nrm}=\mathrm{iso}>$.

31. Mathews I, Pillon S. Factores protectores y de riesgo asociados al uso de alcohol en adolescentes hijos de padre alcohólico, en el Perú. Rev Latino-am Enfermagem. 2004 Mar-Abr; 12 (número especial): 359-68 [Internet] [acceso: 26 de noviembre del 2014]. Disponible en: http://www.scielo.br/pdf/rlae/ v12nspe/v12nspea10.pdf.

32. Calafat Far A. El abuso de alcohol de los jóvenes en España. Adicciones. 2007; 19: 217-23 [Internet] [acceso: 17 de marzo del 2015]. Disponible en: http://www. redalyc.org/articulo.oa?id=289122084001. 